\title{
Smart Robotic Exoskeleton: a 3-DOF for Wrist-forearm Rehabilitation
}

\author{
Noor Sabri Shalal ${ }^{1}$, Wajdi Sadik Aboud ${ }^{2 *}$ \\ ${ }^{1}$ Biomedical Engineering Department, Al-Nahrain University, Baghdad, Iraq \\ ${ }^{2}$ Prosthetics and Orthotics Engineering Department, Al-Nahrain University, Baghdad, Iraq \\ Email: ${ }^{1}$ noorsabri93@yahoo.com, ${ }^{2}$ wajdisadik@gmail.com \\ *Corresponding Author
}

\begin{abstract}
To regain the activities of daily living (ADL) for patients suffering from different conditions such as stroke and spinal cord injury, they must be treated with a rehabilitation process through programmed exercises. The human motor system can learn through motor learning. This study focused on rehabilitating wrist and forearm joints to restore the ADL by designing and constructing a robotic exoskeleton. The exoskeleton was designed to rehabilitate the patients by providing a 3-degree-of-freedom (DOF), including flexion/ extension, adduction/abduction, and pronation/supination movements. It was specified to be portable, comfortable, lightweight, compatible with the human anatomical structure, and provided a speed and range of motion (ROM) as in normal subjects. It was designed with a SolidWorks and constructed with a 3D-printer technique using polylactic acid (PLA) plastic material. The overall exoskeleton was controlled with electromyography; angle information was extracted using EMG MyoWare and gyroscope sensors. It was applied for evaluation using 5 normal subjects and 12 subjects of stroke and spinal cord injury (SCI). As a result, the proposed exoskeleton had a strong impact on regaining muscle activity and increasing the ROMs of wrist and forearm joints. These results prove that the proposed exoskeleton can be used to perform physiotherapy exercises.
\end{abstract}

Keywords-Exoskeleton, Robotic, Wrist, Stroke, SCI, Rehabilitation.

\section{INTRODUCTION}

Muscle weakness and spasticity (resistance to muscle stretch) have significantly impacted activities of daily living (ADL). Several conditions have lead to such effects, including stroke and spinal cord injury (SCI) [1]. Upper limb disorders limited the motions of patients being conditioned with a specific defect, leading to an increase in their dependency and restrict their motions to a limited degree [2].

One of the human's brain properties is self-arrangement. Through an excitation to the afferent and efferent nerves, the neural pathways can re-advancement to regaining the activities of daily living. Since thousands of years ago, rehabilitation has been introduced to recover or enhance the functions of the motor unit. In hospitals and rehabilitation centers, conventional treatment for disabled patients was presented. This sited therapy includes an interaction between the patient and the therapist; therapists guide the patient to perform a repetitive exercise based on a specific program arranged by them $[3,4]$.

The success of such treatment depends on several factors, including the number of repetitive exercises, the period of rehabilitation, condition of patient disability, and date since the disability [5,6]. There are several problems associated with traditional rehabilitation, such as the decrease in therapy time as the number of patients is small compared with the number of therapists. Moreover, the traditional rehabilitation lacked patient progress assessment through their therapy and after it. Due to these limitations, rehabilitation robots have been presented and developed over the years [7].

Rehabilitation robotics is considered as a specific branch of biomedical engineering that roles in decreasing and solving the problems related to traditional rehabilitation. By developing the proposed exercises and evolution of the robotics devices, robotics rehabilitation can support several functions of the sensorimotor. Rehabilitation robotics is subdivided into three main types: upper limbs, lower limbs, and full body. [8-12].

Generally, robotics can be classified into an end-effector and exoskeleton. The end-effector is easy to be implemented and interact with the human at one end, enabling the patient to hold it with his/her hand and provided the motions at the joints. However, it forwards limited information about the patient's limb. On the contrary, the exoskeleton provides a quantitative assessment of the limb. Moreover, the exoskeleton structure has links and joints that similarly match with the human anatomical one. The exoskeleton consists of electronic components, actuators, and controllers. They were reinforced with algorithms to feed the actuators with data, enabling the exoskeleton to work as required [13-16].

Several studies had interested in this field of rehabilitation, aiming to overcome the problems associated with traditional rehabilitation [17-22]. There are several restrictions related to that studies regarding their mode of operations, number ranges of motions. Some of them have designed with 2-DOF or 1-DOF manipulators that may cause ineffective and incomplete rehabilitation therapy, making the joint regain its activity in two or one motion rather than of all them. Other studies were interested in the haptic part of the device rather than overall construction design and technology. Moreover, some of them had hardware complexity with stationary conditions and high cost, making the rehabilitation therapy available only in hospitals and rehabilitation centers. Furthermore, the studies were only restricted to specific defect conditions. These studies have not mentioned any trial with patients with a 
defective condition except in [22], which applied their device to incomplete SCI patients with 10 sessions.

The objective of this study is to overcome the problems associated with those studies mentioned above in this field. This study aims to design, construct and implement a wearable, low-cost, 3D printed forearm-wrist robotic exoskeleton controlled using sEMG signal assisted with angle sensor by providing the exercises for rehabilitation of patients who suffer from stroke and spinal cord injury. This therapy is commonly used in restoring lost motor skills by helping the brain rebuild neural pathways lost due to disease or trauma such as stroke. In removing the need for a physical therapist to conduct these exercises, the patients would be able to devote more time to their therapy at a lower cost while achieving a greater level of independence. In addition, a home-based therapy without the need for significant supervision can be done. This can lead to rapidly progressing in the rehabilitation process in centers and hospitals that have more patients than the number of physiotherapists.

\section{ANATOMY AND BIOMECHANICS}

Two bones that constitute the forearm are called the radius and ulna. The forearm is considered a tri-articular structure that connects the wrist and elbow joints. An interosseous membrane is a structure between radius and ulna which intercalated between (PRUJ) and (DRUJ), providing a mid-radioulnar joint (MRUJ). The pronation/supination movement of the forearm is achieved by rotating the radius around the ulna [23], as shown in Fig. 1. Several muscles are included in the posterior and anterior compartments of the forearm responsible for movements of the wrist, hand, and elbow joint [24]. At the wrist joint, the ulna bone conveys approximately $20 \%$ of the load-bearing force, and the radius bone translates the remaining $80 \%$. While the humeroulnar joint carries almost $43 \%$ of the load at the elbow joint, the $57 \%$ remaining load is transmitted by the humeroradial joint. The wrist joint is a sophisticated musculoskeletal joint. Several structures are congregated to form such joint, including the distal end of ulna and radius, the proximal end of metacarpal bones, the proximal and distal rows of eight bones known as carpal bones [25]. The wrist joint can move in the sagittal (flexion/extension) and frontal (adduction/abduction) planes (see Fig. 1). The ligamentous and bony structure of the wrist permits it to hold a load 10 times larger than the load that can keep the fingertip through the grip [26].

\section{DESIGN CONSIDERATION}

As the device is based on repetitive training of patients to restore their activities and intact with them, it must meet several requirements: 1) Kinematic: it must meet that of the destined joint, 2) Safety: it must be as safe as possible. It must be reinforced with precise controlling, mechanical stoppers, an accurate component to prevent hyper movement giving accurate measuring parameters, 3) Comfortability: the device must be adjustable, fitting to various patients regardless of size, shape, age, and volume of their lower arm, 4) Structural mechanism: the exoskeleton must not be bulky to allow the patient to move freely without resistance, and its structural mechanism should be meet that of the human ones. All of these requirements have been achieved in the proposed exoskeleton.
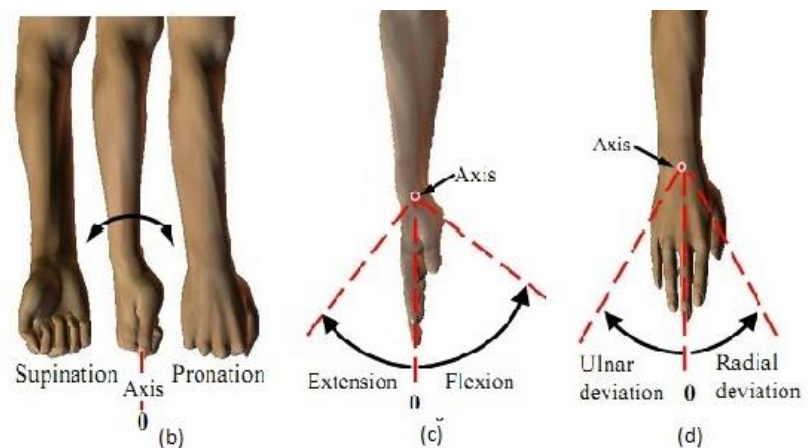

(d)

Fig. 1. Kinematics of Forearm and Wrist [27]

\section{MECHANICAL DESIGN}

The robotic exoskeleton was designed to meet the destination requirements based on the biomechanical, anatomical, and anthropometric parameters. The robotic exoskeleton is divided into two main parts. The first part is the wrist design which is divided further into two parts. The second part is the forearm part designed to provide a movement in one anatomical reference plane. These parts were designed using the 3D CAD software SOLIDWORKS and printed using 3D printer technology using polylactic acid (PLA). Fig. 2 shows the CAD model of robotic exoskeleton parts and their assembly. Moreover, these parts are designed to be compatible for all people regardless of sex, age, length, and weight.

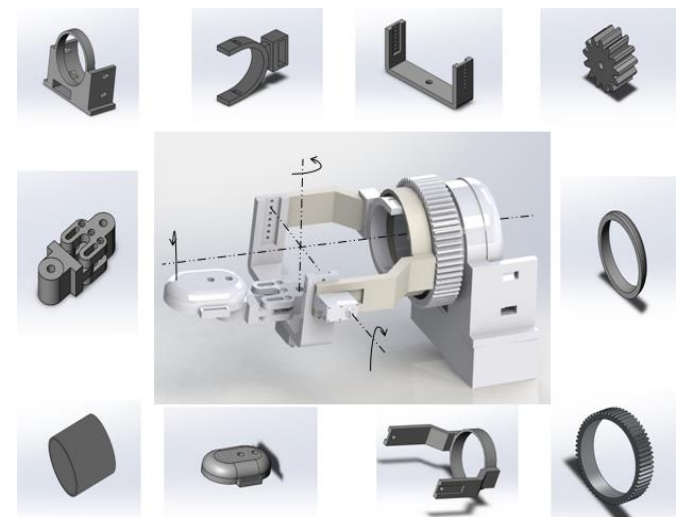

Fig. 2. CAD Model of the Exoskeleton Parts and its Assembly.

The forearm design, as shown in Fig. 3, consists of several components, including cylinder structure, gears, wrist holder, circular fastener structure, and the structural mechanism. The forearm design represents the pronation/supination that provides the same range of motion as in an average human. The forearm part provides the pronation and supination movement through an actuation using DS3218 Digital servo motor. Two gears have been used as a power transmission method allowing the translation of the movement from bevel gear to spur gear, resulting in the rotation of the forearm mechanism. 


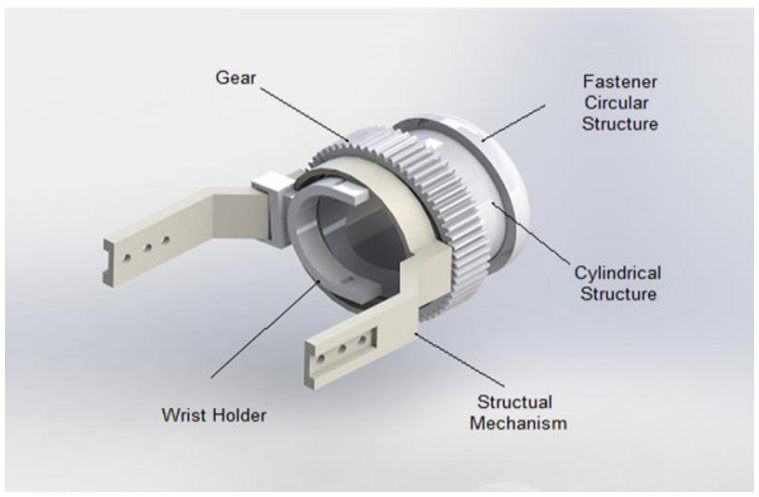

Fig. 3. Forearm Design.

The wrist design consists of the flexion/extension part and adduction/ abduction part shown in Figs. 4 and 5, respectively. The flexion/extension part reflects the flexion/extension range movement of the exoskeleton as in the average human. This movement was achieved using DS3218 Digital servo motor placed at the contact point between the flexion/extension part and the distal end of the mechanical structure of the forearm mechanism being attached screw. Meanwhile, the adduction/abduction part ( see Fig. 5) was represented by the distal part of the exoskeleton that consists of two mechanical structure attached through springs. Thus, it provided a flexible and unconstrained motion. The distal part also includes two handle mechanisms that were used according to the rehabilitation progress. The first mechanism was used during the passive mode of operation (the patient has no muscle activity), which has a strip being passed through its end-sides, holding the hand. The second mechanism was used during the active mode (the patient exhibits some muscle activity). The adduction/abduction part provided the movement using Tower Pro mg995 servo motor connected to the flexion/extension part through a screw.

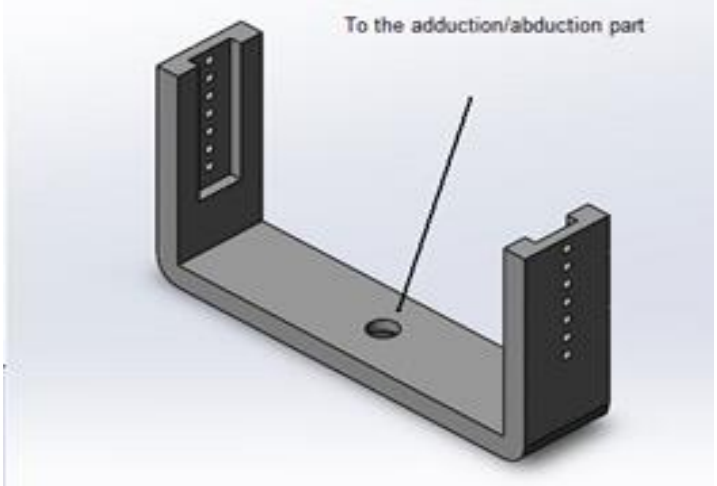

Fig. 4. Flexion/Extension Part.

Mechanical stoppers were designed and placed at specified locations in the forearm and wrist to restrict the movements of the servo motors. They functioned to prevent the excesses motions and follow the normal human movement (the ROM of flexion/ extension, adduction/ abduction, and pronation/supination parts to $130^{\circ}, 70^{\circ}, 150^{\circ}$, respectively). Thus, a safer system could be provided. Fig. 6 and 7 show the final structure of the exoskeleton compatible with the electronic circuit.

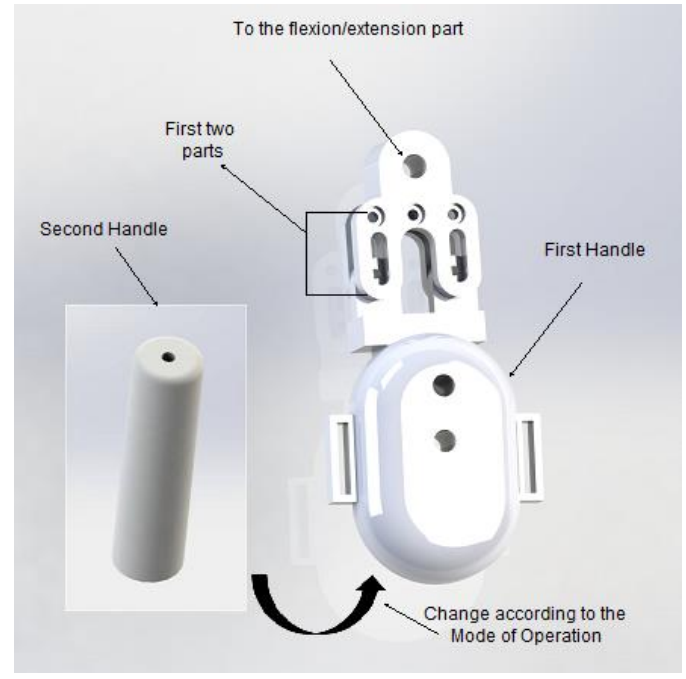

Fig. 5. Adduction/Abduction Part.

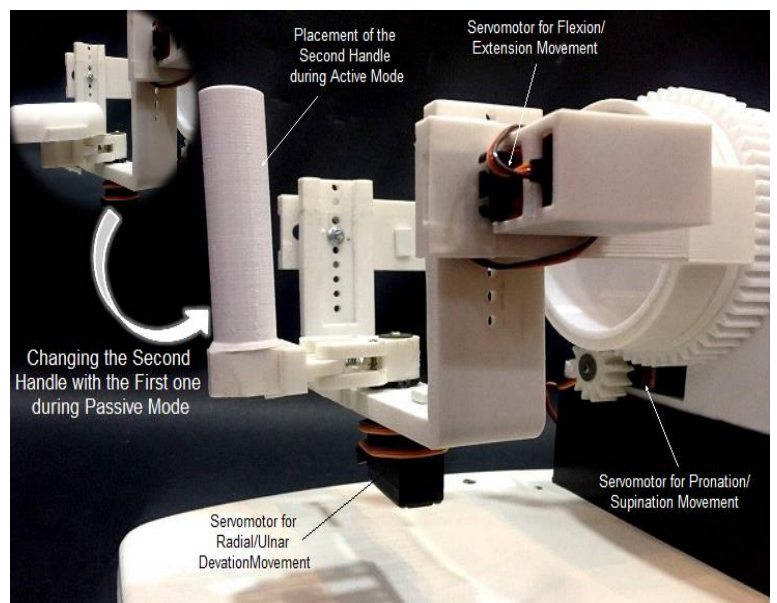

Fig. 6. Servomotors Placements.

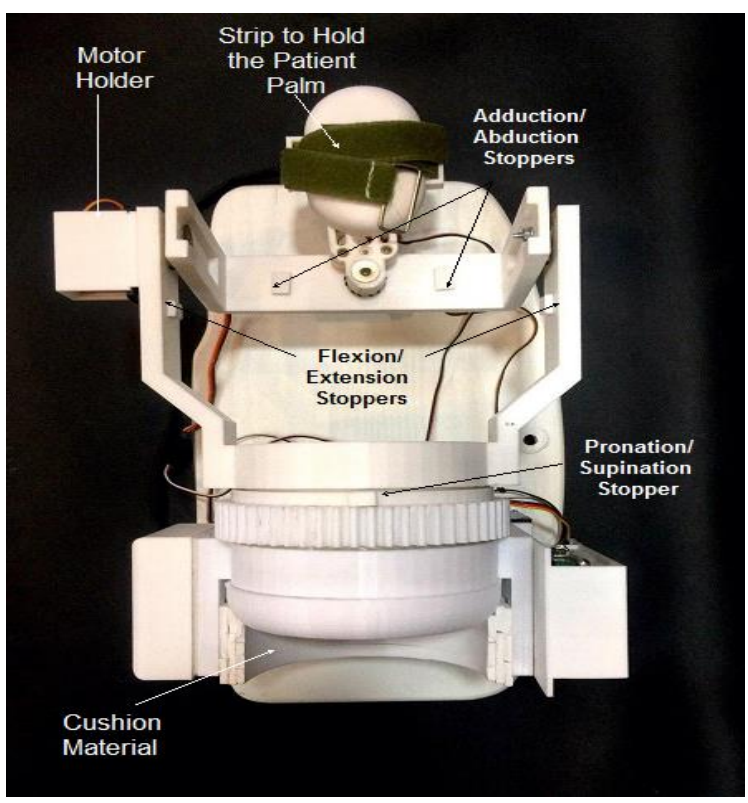

Fig. 7. Final Assembly of the Exoskeleton 


\section{ExOSKeleton Manufacturing Process}

All constructed parts of the exoskeleton were built and configured by an additive technique called $3 \mathrm{D}$ printing. It is characterized by its naivety and customization, in addition to its low-cost manufacturing. The exoskeleton was made using the Fused deposition molding technology (FDM). A filament or metal wire was released from a coil to prepare an extrusion nozzle. One of the characteristics that must be noted is that the printed parts were very strong along the printing plane than the normal ones. During the printing process, each heated layer was met with the next layer and drawn together. Once the subsequent layer is printed, the former will be cooled down and hardened [28].

\section{ELECTRONIC DESIGN}

\section{A. Materials}

Several electronic components were used for this study, including EMG MyoWare, gyroscope sensors, pushbuttons, buzzer, microcontroller, V2.0 shield, and liquid crystal display (LCD). An EMG MyoWare sensor from Advancer Technologies was used to measure muscle activity noninvasively. Two EMG MyoWare sensors were also placed on the extensor carpi ulnaris and pronator teres, as shown in Fig. 8 .
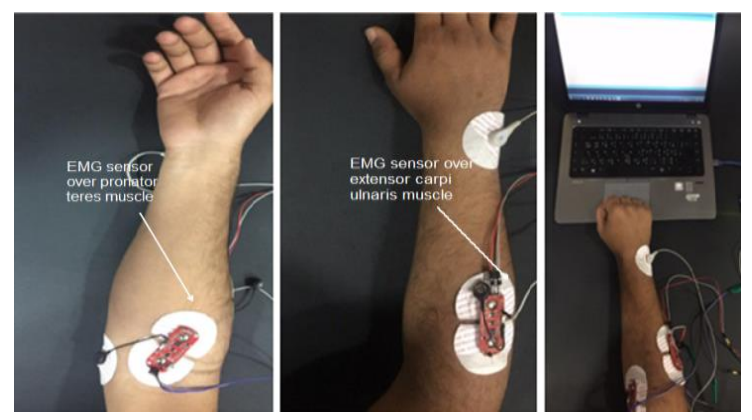

Fig. 8. Placement of EMG MyoWare Sensor.

A gyroscope sensor was used to measure the range of motion (ROM) and angular velocity around three axes for three proposed movements, as illustrated in Fig. 9. Both the EMG sensors and gyroscope sensors were used to provide the signal for controlling servo motors to achieve the required movements and evaluate the rehabilitation progress. Fig. 9 shows the use of a gyroscope sensor for angle measurement.

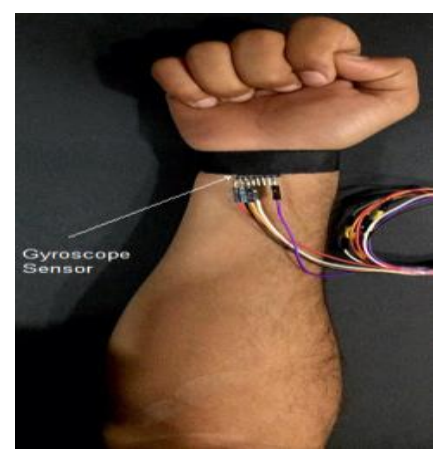

Fig. 9. Angle Measurement with Gyroscope Sensor.
Buzzer and LCD were used as an indicator for translation between movements and cases. They were also used to display the angle readings. Pushbuttons were used as a switch to different operational modes and cases.

\section{B. Control system}

In this system, an EMG MyoWare, angle sensors, and switches were utilized to control the overall system. An EMG MyoWare sensors extracted the muscle activity from the targeted muscles. Then, these data were used to control the 3DOF movements of the system's servo motors. The controlling process was done by determining the specified threshold value for both muscles. In addition, the gyroscope sensor was also used in controlling the process of this system. The servo motors were programmed to restrict the reflected range of motions to a specified range as in normal humans. Mechanical stoppers were used to prevent the excessive motions reflected by these motors as mentioned in the mechanical design section. The switches were used to control the interchangeable processes between different modes of operation that can be chosen according to the patient's rehabilitation progress and his/her conditions. The proposed modes of operation were passive mode and active mode.

\section{RESULTS}

The exoskeleton was checked alone for its reflected range of motions, velocities, motors bearing torque, prolonged working time, and battery lifetime. Then, the device was tested on normal subjects of different ages, sex, and weight. The exoskeleton's kinematic, working, bearing, comfortability, safety, and efficiency were achieved before tested on patients. Figures (10-15) and Table 1 show the range of motion and angular velocity analysis of the robotic exoskeleton for average human.

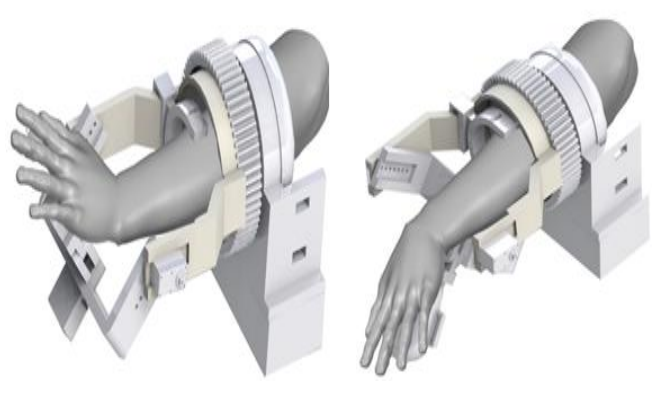

Fig. 10. Extension and Flexion Movement of the Exoskeleton.

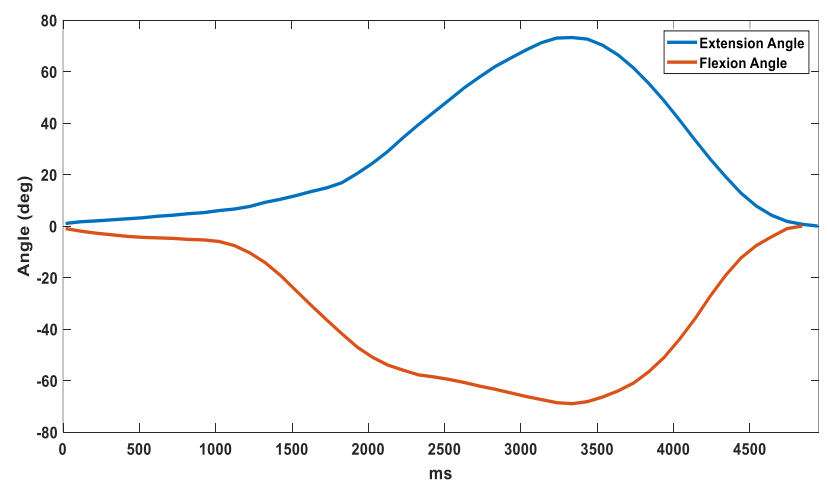

(a) 


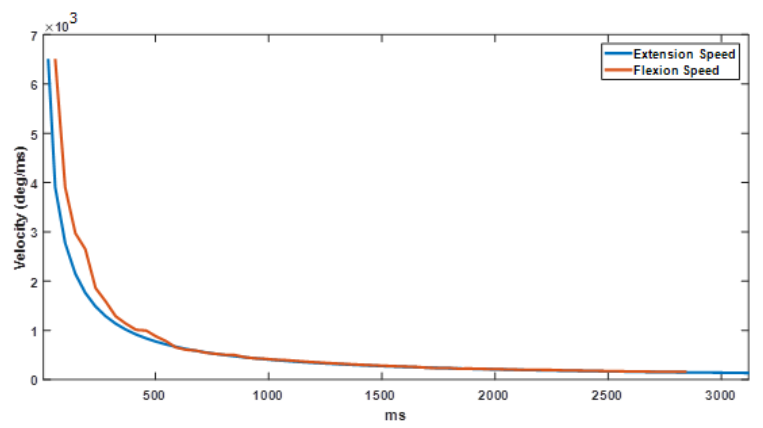

(b)

Fig. 11. ROM (a) and Velocity (b) Flexion/Extension

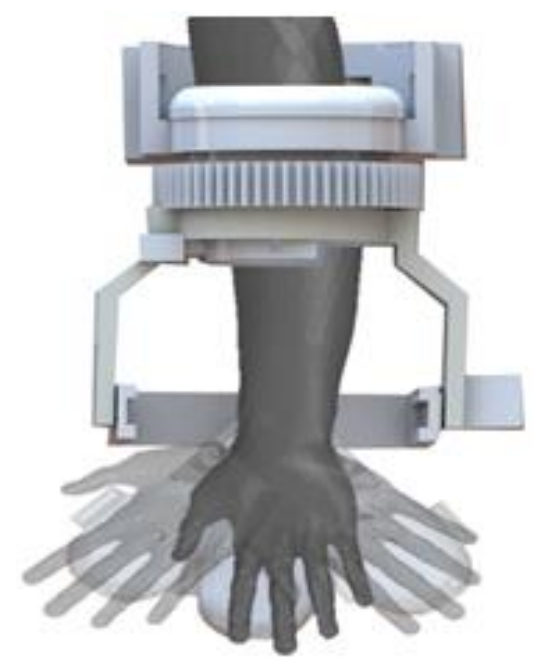

Fig. 12. Adduction and Abduction Movement of the Exoskeleton.

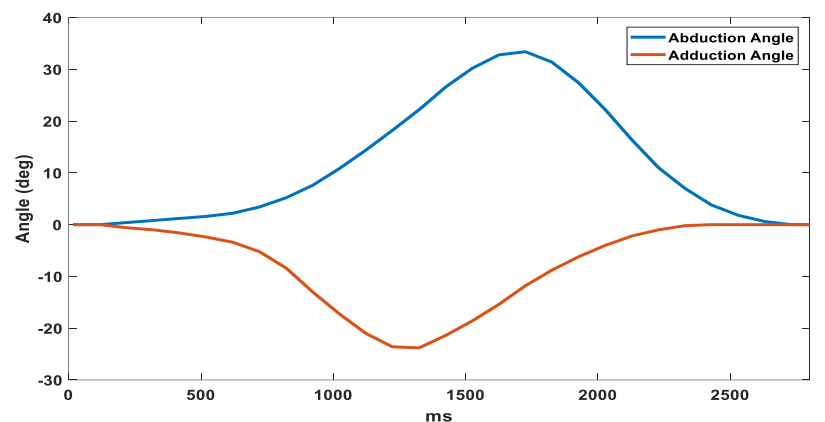

(a)

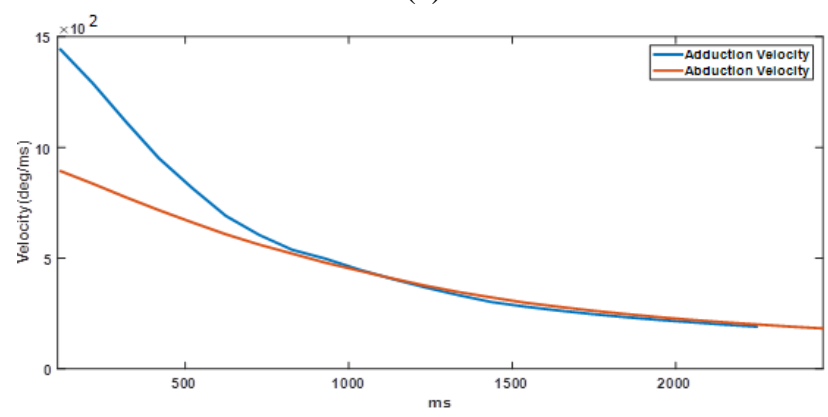

(b)

Fig. 13. ROM (a) and Velocity (b) Analysis of the Adduction/Abduction Movement of the Exoskeleton

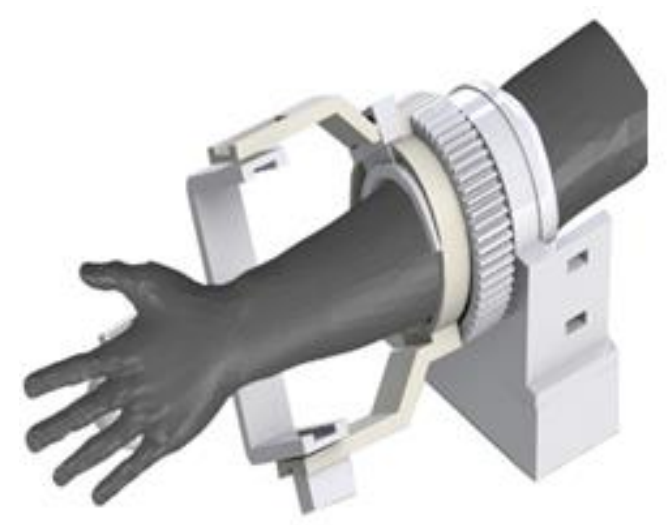

Fig. 14. Rotational Movement of the Exoskeleton.

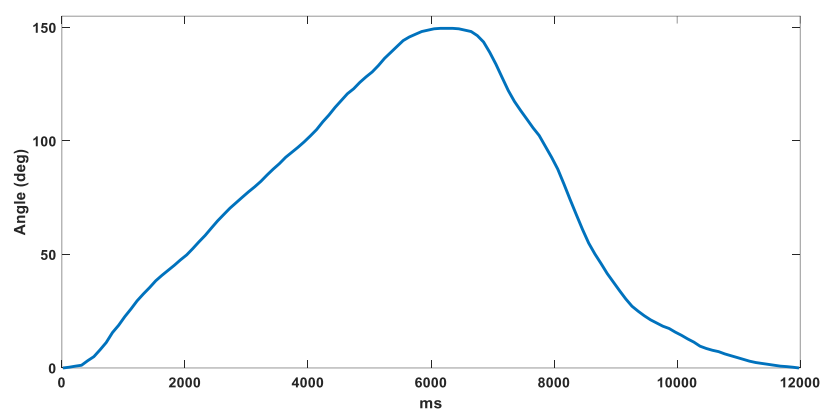

(a)

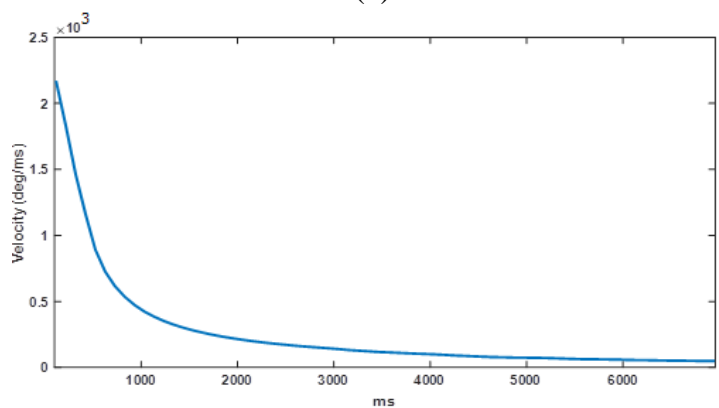

(b)

Fig. 15. ROM (a) and Velocity (b) Analysis of the Rotational Movement of the Exoskeleton.

TABLE I. ROMS AND VELOCITY INFORMATION OF THE NORMAL HUMAN

\begin{tabular}{|l|l|l|}
\hline \multicolumn{1}{|c|}{ Type of motion } & \multicolumn{1}{|c|}{$\begin{array}{c}\text { Normal angle } \\
(\mathbf{d e g})\end{array}$} & $\begin{array}{c}\text { Normal Velocity } \\
(\mathbf{d e g} / \mathbf{s})\end{array}$ \\
\hline Pronation & $80^{\circ}$ & 803.548 \\
\hline Supination & $70^{\circ}$ & \\
\hline Flexion & $75^{\circ}$ & 1970.168 \\
\hline Extension & $70^{\circ}$ & 2175.768 \\
\hline Adduction & $25^{\circ}$ & 27496.58 \\
\hline Abduction & $35^{\circ}$ & 22955.46 \\
\hline
\end{tabular}

After examining its required speed and ROMs, the proposed exoskeleton was applied to stroke and spinal cord injury patients. Table 2 shows the information of the patients. 
TABLE II PATIENT'S INFORMATION

\begin{tabular}{|l|l|l|}
\hline Data & R. H. & M. A. \\
\hline Age & 49 & 52 \\
\hline Gender & Male & Male \\
\hline Affected Arm & Right & Right \\
\hline Type of Defect & Incomplete SCI & Stroke \\
\hline Length $(\mathrm{mm})$ & 175 & 176 \\
\hline Weight $(\mathrm{Kg})$ & 75 & 120 \\
\hline
\end{tabular}

Figures (16-18) show the EMG activities and ROMs for wrist and forearm joints in the first, fourth, and seventh sessions of the stroke patient.

The rehabilitation process began with a passive exercise in the first session. Then, the active exercises were started in the fourth session. The last session indicated the physiotherapy progress, which slightly approximated the normal human parameters. This program was applied to a stroke patient.

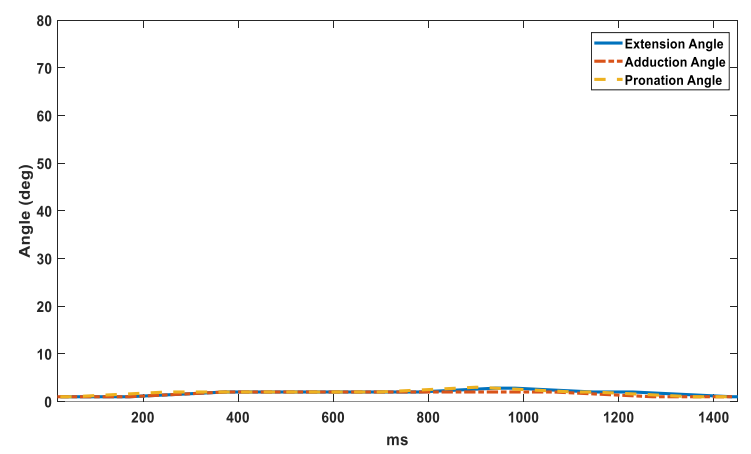

(a)

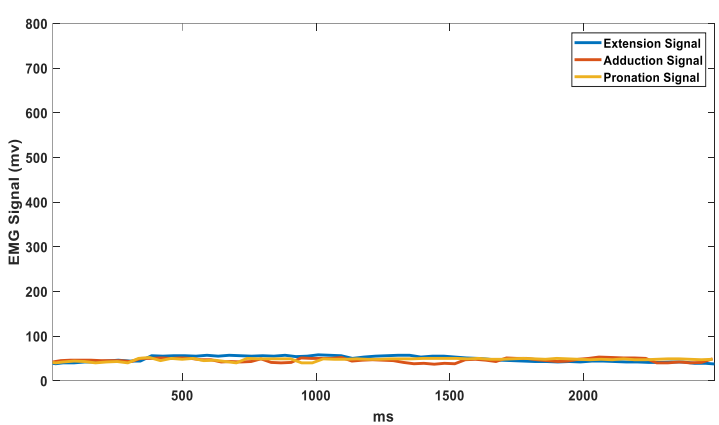

(b)

Fig. 16. ROMs (a) and Muscle Activities (b) of Stroke Patient in $1^{\text {st }}$ session.

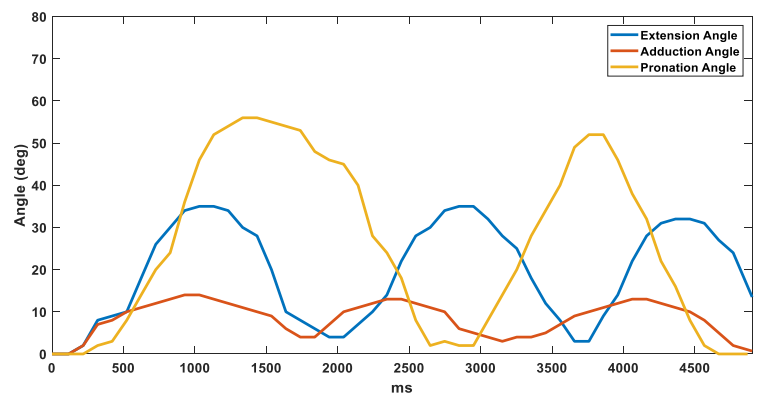

(a)

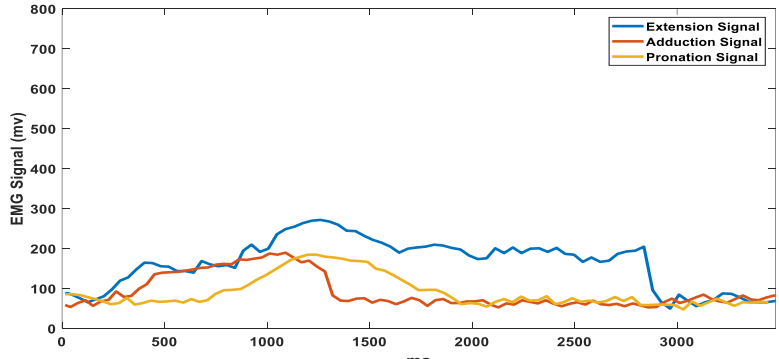

(b)

Fig. 17. ROMs (a) and Muscle Activities (b) of Stroke Patient in $4^{\text {th }}$ session.

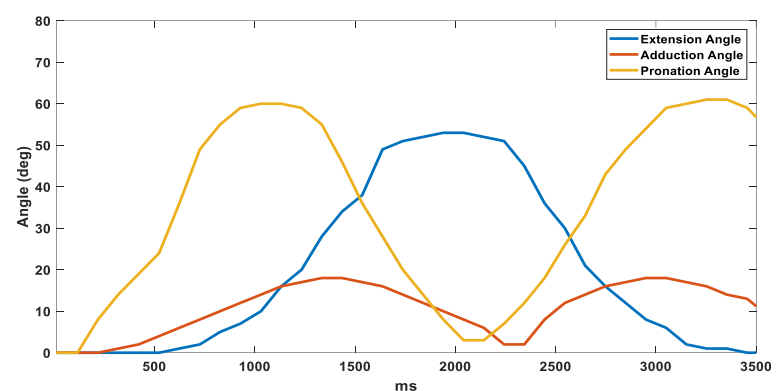

(a)

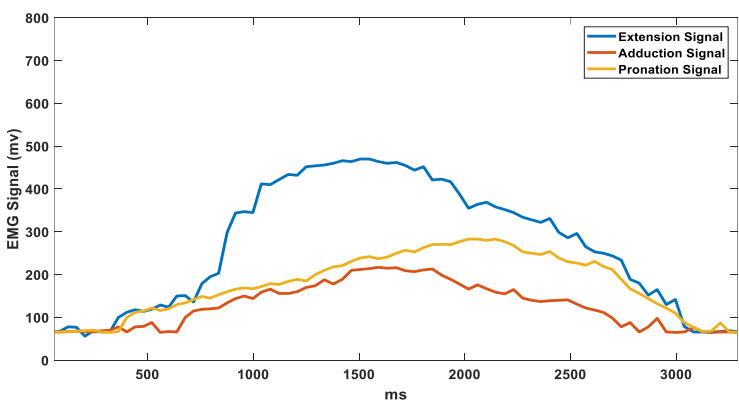

(b)

Fig. 18. ROMs (a) and Muscle Activities (b) of Stroke Patient in $7^{\text {th }}$ session.

Meanwhile, for incomplete SCI patients, the rehabilitation process was started with active exercises. Then, the rehabilitation progress was recorded throughout sessions until the muscular activities and ROMs were achieved approximately as in normal subjects.

Figures (19-21) present the EMG signal and ROMs progress throughout the proposed sessions.

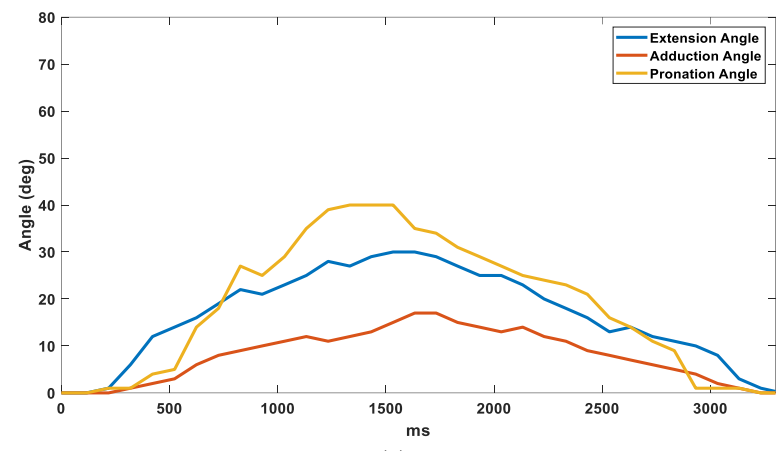

(a) 


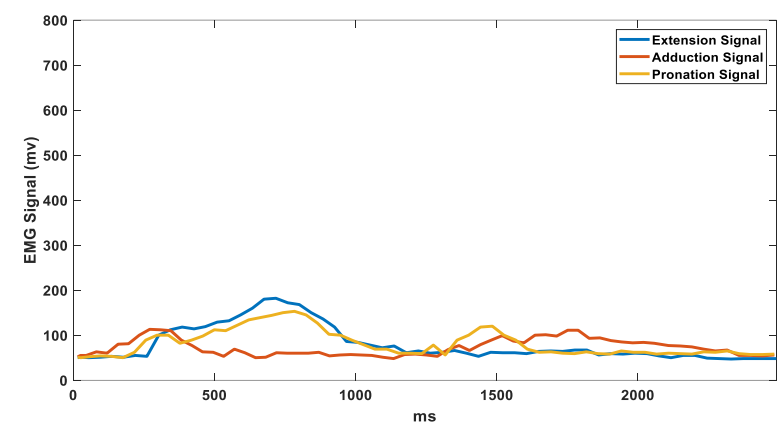

(b)

Fig. 19. ROMs (a) and Muscles Activities (b) of SCI Patient in $1^{\text {st }}$ session.

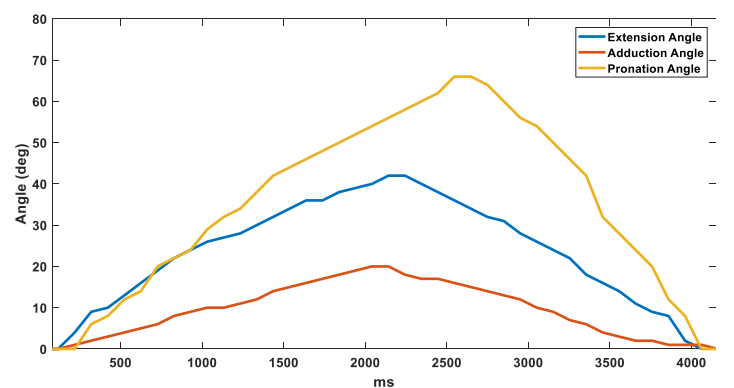

(a)

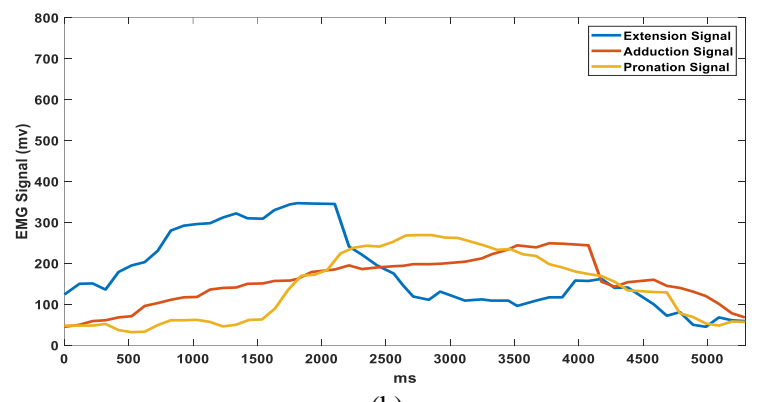

(b)

Fig. 20. ROMs (a) and Muscles Activities (b) of SCI Patient in $5^{\text {th }}$ session.

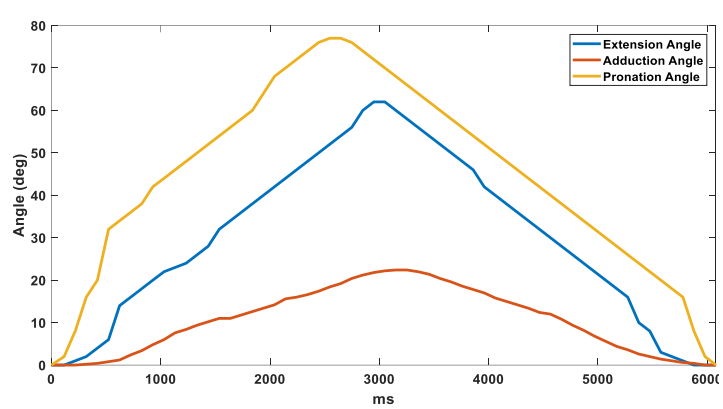

(a)

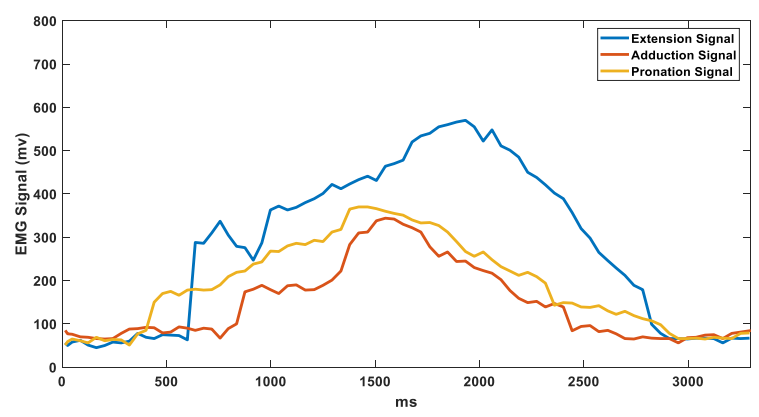

(b)

Fig. 21. ROMs (a) and Muscles Activities (b) of SCI Patient in $9^{\text {th }}$ session.

\section{VIII.CONCLUSION}

Impairment of the upper limb's motor function is a series of problems due to their effectiveness in inhibiting the activities of daily living. The rehabilitation program in this study was started with passive and active exercises for stroke patients and active exercise for SCI patients. It can be concluded that the use of EMG signal and gyroscope sensors is of utmost importance for the evaluation of the rehabilitation process and controlling strategies of the exoskeleton. The EMG and gyroscope sensors were not used during passive exercises. As the patient reached or exhibited muscular activity and ROMs progress, the rehabilitation process was translated to active exercises. It was found that the EMG signal and ROMs for 3DOF were enhanced while using the exoskeleton as a rehabilitation device. Thus, it can be concluded that the exoskeleton can be an alternative in rehabilitation. It could minimize the work of physiotherapists and has low price, reduces rehabilitation time with a large enhancement of progress. Eventually, it can restore the activities of daily living of patients as nearly for normal subjects as possible.

\section{ACKNOWLEDGMENT}

The authors acknowledge collaborators whose work either influenced or was explicitly incorporated into this article. Special thanks to the staff of medical instruments and prosthetics and Orthotics Laboratories at the College of Engineering / Al-Nahrain University for their support and technical help.

\section{REFERENCES}

[1] Zeiler S.R., and Krakauer J.W., "The interaction between training and plasticity in the poststroke brain", Curr Opin Neurol, 26(6), pp. 609$16,2013$.

[2] O'Sullivan S., Schmitz T.J., and Fulk G., "Physical rehabilitation",(F.A. Davis Co., PA), 6th ed., pp. 664-667, 2014.

[3] Krebs, H. I., et al., "Robot-Aided Neurorehabilitation: A Robot for Wrist Rehabilitation", IEEE Transactions on Neural Systems and Rehabilitation Engineering, 15(3), pp. 327-335, 2007.

[4] Mauk K. L., "Overview of rehabilitation", Rehabilitation Nursing: A Contemporary Approach to Practice, 2011.

[5] Feys H., et al., "Early and repetitive stimulation of the arm can substantially improve the long-term outcome after stroke: a 5-year follow-up study of a randomized trial", stroke, 35(4) pp. 924929,2004. doi:10.1161/01.str.0000121645.44752.f7.

[6] Patton J., Small S.L., and Rymer W.Z., "Functional restoration for the stroke survivor: informing the efforts of engineers", Top Stroke Rehabil,15(6) pp. 521-541,2008. doi:10.1310/tsr1506-521.

[7] Yap H. K., et al., "Design and Preliminary Feasibility Study of a Soft Robotic Glove for Hand Function Assistance in Stroke Survivors", Frontiers in Neuroscience, 11, p. 547, 2017. doi:10.3389/fnins.2017.00547, 2017.

[8] Gopura R. A. R. C., Kazuo K., Bandara D. S. V., "A brief review on upper extremity robotic exoskeleton systems", 6th International Conference on Industrial and Information Systems, ICIIS, Sri Lanka, 2011.

[9] Rehabilitation robotic, retrieved from Wikipedia, available on https://en.wikipedia.org/wiki/Rehabilitation_robotics

[10] RewerBambi R., McDowell S.K., and Worthen-Chaudhari L. C., "Poststroke Upper Extremity Rehabilitation: A Review of Robotic Systems and Clinical Results", Topics in Stroke Rehabilitation, 14 (6), pp. 22-44, 2007. doi:10.1310/tsr1406-22. PMID 18174114.

[11] Marchal-Crespo L., and Reinkensmeyer D. J., "Review of control strategies for robotic movement training after neurologic injury", Journal of NeuroEngineering and Rehabilitation, pp. 6- 20, 
2009. doi:10.1186/1743-0003-620. PMC 2710333. PMID 19531254.

[12] Senailayake C., and Senanayake S., "Emerging robotics devices for therapeutic rehabilitation of the lower extremity," in Advanced Intelligent Mechatronics,IEEE/ ASME International Conference on. IEEE, pp. 1142-1147, 2009.

[13] Francesco A., Tadeusz M., and James O., "Robotics for rehabilitation of hand movement in stroke survivors", Advances in Mechanical Engineering, 11(4), pp. 1-14, 2019.

[14] Stephen H. S., and Sean P D., "Potential of robots as next-generation technology for clinical assessment of neurological disorders and upper-limb therapy", The Journal of Rehabilitation Research and Development, 48( 4), pp. 335-354, 2011.

[15] Baldovino R. G., and Jamisola R. A., "A Study O The State Of Powered-Exoskeleto Design For Lower Extremities", In 5th International Conference on Humanoid, Nanotechnology, Information Technology, Communication and Control, Environment, and Management, 2009.

[16] Ferris D. P., Young A. J. , "in Encyclopedia of Biomedical Engineering", Elsevier, Imprint Elsevier, 2019.

[17] Zeeshan O. K,, Zhen G, X., and Carlo M., "Surface EMG pattern recognition for real-time control of a wrist exoskeleton",BioMedical Engineering OnLine, 9, p.41, 2010.

[18] Omarkulov N., et al.," Preliminary mechanical design of NU-Wrist: A 3-DOF self-aligning Wrist rehabilitation robot", 6th IEEE International Conference on Biomedical Robotics and Biomechatronics (BioRob), (pp. 962-967),2016.

[19] Al-Fahaam H., Steve D., and Samia N., "A novel, soft, bending actuator for use in power assist and rehabilitation exoskeletons" ,
IEEE/RSJ International Conference on Intelligent Robots and Systems (IROS), pp. 533-538,2017.

[20] Lambelet C., et al.," The eWrist - a wearable wrist exoskeleton with sEMG-based force control for stroke rehabilitation" International Conference on Rehabilitation Robotics (ICORR), (pp. 726-733), 2017.

[21] Ikuo Y., et al., "Wrist Rehabilitation Robot System and Its Effectiveness for Patients", Sensors and Materials, 30(8), 2018.

[22] Kadivar Z., et al.," Robotic training and kinematic analysis of arm and hand after incomplete spinal cord injury: A case study", in 2011 IEEE International Conference on Rehabilitation Robotics.

[23] European Federation of National Associations of Orthopaedics and Traumatology, Vol.13, 2013.

[24] Hsu H., and Siwiec R. M., "Forearm Splinting", StatPearls PUBLISHING, 2019.

[25] Author, "Applied anatomy of the wrist, thumb and hand", Elsevier, Ltd, 2013.

[26] Kamal R. N., Starr A., and Akelman E., "Carpal Kinematics and Kinetics", _Elsevier, 2016.

[27] Gopura, R. A. R. C., et al., "Developments in hardware systems of active upper-limb exoskeleton robots: A review", Robotics and Autonomous Systems, 75, 203-220, 2016.

[28] Leal J A, Rene C, Miguel S, Remero M F and Saez L M," Structural numerical analysis of a three fingers prosthetic hand prototype,", pp. 526-536 academic journal, España April 2013. 